\title{
With whom to merge? A tale of the Spanish banking deregulation process
}

\author{
Ana Lozano-Vivas • Miguel A. Meléndez-Jiménez • \\ Antonio J. Morales
}

Received: 18 March 2010 / Accepted: 22 November 2010 / Published online: 14 December 2010 (C) The Author(s) 2010. This article is published with open access at SpringerLink.com

\begin{abstract}
We propose a spatial competition model to study banks' strategic responses to the asymmetric Spanish geographic deregulation process. We find that once the geographic deregulation process finishes, inter-regional mergers between savings banks are optimal whenever the economies of scale associated to merging activities are low. If there are large gains, then there will be mergers between savings and commercial banks.
\end{abstract}

Keywords Branch deregulation - Mergers · Optimal behavior - Spanish Banking System

JEL Classification $\quad \mathrm{C} 72 \cdot \mathrm{G} 21 \cdot \mathrm{G} 28 \cdot \mathrm{L} 13 \cdot \mathrm{L} 41 \cdot \mathrm{L} 51$

\section{Introduction}

The current world economic crisis has led to many debates throughout the world, the debates being necessarily more heated for those countries experiencing deeper (and longer-lasting) recessions. This is the case of Spain, where a far-reaching comprehensive structural reform is needed. In particular, even though the Spanish banking

We are thankful to two anonymous referees, and to María Ángeles de Frutos (the Editor in charge), whose detailed comments helped us to improve the quality of the manuscript and to shape the exposition. We gratefully acknowledge financial support from the Spanish Ministry of Education (project ECO2008-04424) and from the Andalusian High Excellence Research Program (project SEJ 4794).

A. Lozano-Vivas · M. A. Meléndez-Jiménez · A. J. Morales ( $\varangle)$

Facultad de Ciencias Económicas y Empresariales, Universidad de Málaga,

Plaza El Ejido s/n, 29013 Málaga, Spain

e-mail: amorales@uma.es

A. J. Morales

LINEEX, Universidad de Valencia, Campus Tarongers, 46022 Valencia, Spain 
sector (SBS) has not (yet) contemplated the bankruptcy and intervention of any major bank, many clamor for structural reforms in the banking system. Such a reform could take the form of a consolidation process, in which the weakest institutions merge to strengthen the system's soundness and efficiency. ${ }^{1}$

There are two major types of institutions in the SBS: savings banks and commercial banks. Historically, commercial banks were created as for-profit limited firms with the aim of performing a whole range of activities related to the provision of financial services. Savings banks, however, started to emerge in the middle of the nineteenth century and they were created as not strictly, or not exclusively, profit-maximizing institutions, and their activity was initially oriented toward families and small businesses. $^{2}$

In the case of Spain nowadays, the weakest banking institutions are mainly savings banks. This is due not only to large exposures to bad loans in the last few years, but also to a quick business switch from very traditional retail banking to more sophisticated product activities, such as securitization and mortgages. ${ }^{3}$ This reorientation started in the late 1980s, when the deregulation process culminated and both commercial and savings banks could freely compete on prices and services. Until then, commercial banks traditionally specialized in wholesale banking, whereas savings banks were more oriented toward retail banking. ${ }^{4}$

The view expressed by the governor of the Bank of Spain, quantifying the magnitude of the reform needed in the Spanish savings banks segment - a third of the 45 unlisted regional savings banks are to be absorbed by stronger institutions - is actually shared by many (See "Bank of Spain chief in reform plea," Financial Times, February 24, 2010). However, despite this consensus, the consolidation process has been slow.

But the problem is not only that the current consolidation process is slow. Historical data confirm the null presence of inter-regional mergers among savings banks since the end of the 1980s, when geographic restrictions on the expansion of these banking institutions were lifted. Also, barriers for mergers between savings and commercial banks currently exist. This paper sheds some light onto the features of the consolidation process prompted by a regime shift. We are interested in analyzing the strategic incentives to compete and merge provided by geographic deregulation.

\footnotetext{
${ }^{1}$ Actually, concentration-stability theory states that concentration sometimes should enhance bank stability, although it is also true that some other times competition is needed to make the industry efficient, profitable, as well as stable (Allen and Gale 2000, 2004; Hellman et al. 2000; Besanko and Thakor 1993; Boot and Greenbaum 1993, and Matutes and Vives 2000; among others). For instance, Beck et al. (2006) find empirical results consistent with concentration-stability theory for a cross-country analysis on 69 countries and 47 crisis episodes.

${ }^{2}$ Savings banks pursue a dual purpose; they are "dual bottom line institutions" that seek both profit and social objectives. The relative weights of the two objectives have changed over time. Due to the stronger competitive challenge that results from the financial liberalization process, at this time the financial objective has more weight. This is required to secure institutional survival, a precondition for providing socially relevant services. See, for instance, Fuentelsaz et al. (2006).

${ }^{3}$ Cuñat and Garicano (2009) provide some explanations about the actual debate on the causes of the weak performance of Spanish savings banks.

${ }^{4}$ For a detailed overview of the Spanish banking deregulation process, see, for instance, Caminal et al. (1989), Gual and Neven (1992), Canals (1997), Carbó (2004), Salas and Saurina (2003), and Fuentelsaz et al. (2006), among others.
} 
Examinations of the consequences of geographic regulation on bank behavior from an empirical point of view abound. The usual finding is that economic and bank performance and efficiency improved once geographic restrictions were lifted. ${ }^{5}$ However, despite the fact that the regulation of geographic expansion was common to all countries, only a few papers have theoretically examined the interplay between geographic regulation and banking behavior. Economides et al. (1996) rationalize the geographic restrictions as an effective means of protecting small banks from large ones in the United States, and Lozano-Vivas et al. (2010a) analyze the consequences of removing these barriers in the United States.

This paper attempts to contribute to the theoretical literature that relates geographic regulation and banking behavior by proposing a spatial competition model, based on Salop (1979), with which to study banks' strategic responses to geographic deregulation. The main strategic variable we consider is a quantity variable: branching. ${ }^{6} \mathrm{We}$ are interested in analyzing asymmetric geographic regulation, that is, when regulation is not uniform across different types of banking institutions belonging to the same banking system. ${ }^{7}$ We consider two unequal territories (allowing for wealth differences across territories) and three banking institutions: one multimarket bank, free to open branches in every territory, and two local banks, one in each territory, restricted to open branches in their local territories. The deregulation game is modeled as a standard incumbent-entrant model in which initial conditions are determined by the asymmetric regulatory body. Once geographic deregulation is announced, incumbents in each territory have the opportunity to resize their branch network before entry decisions are taken.

We find that entry occurs in equilibrium, although on a scale that is not large enough to offset the advantage of incumbents in terms of established branches (most of these branches opened during the regulated period). As a result, the multimarket bank, which was incumbent in both territories, becomes the largest bank. The two local banks come next, with the incumbent in the wealthier territory preceding the incumbent in the poorer one.

This equilibrium configuration is taken as the initial condition for a merger game that assumes that banks are free to maintain merger negotiations and that the surplus of a merger is divided according to the profitability of the two institutions. Given that all mergers provide a positive surplus, it follows that there will be a merger in equilibrium. As regards the identity of the merging banks, it is interesting to note

\footnotetext{
5 Calomiris (2000) suggests that geographic restrictions were the most important factor in creating smaller banks, inhibiting diversification and generating banking instability, making banks more vulnerable to economic downturns, portfolio shocks, and bank runs. On the other hand, based on geographic restrictions in United States, Jayaratne and Strahan (1996, 1998), Kroser and Strahan (1999), and Stiroh and Strahan (2003) state that branching restrictions have benefited smaller, less efficient banks by providing protection from the competition of larger and more efficient firms that would enter the sector were the barriers to entry lifted. In addition, the findings of Amel and Liang (1992), Calem (1994), and Mclanghin (1995) suggest that significant numbers of entries took place following the lifting of geographic restrictions.

6 For tractability, we dispense with price decisions. The main effect is that there will be a one-to-one correspondence between size (measured in terms of the branch network), total assets, and profits in equilibrium. Hence, the most profitable bank will be the largest one.

7 The companion paper of Lozano-Vivas et al. (2010a) deals with the case of symmetric geographic regulation.
} 
that in the determination of the target of a given institution, a tension arises between (i) the largest surplus that can be obtained from merging with a stronger rival and (ii) the higher share of the surplus that can be obtained by merging with a weaker rival.

When the economies of scale are low, this tension is resolved in terms of the surplus share. Accordingly, banks will target to their smaller rival and no bank will therefore target the multimarket bank, which will not be involved in the equilibrium merger. In this case, a merger provides a benefit to the merging institutions, but the non-merging institution benefits the most. The multimarket bank can benefit from this positive externality and manages to stand alone. However, when large gains in scale economies are associated with merger activities, all banks are interested in merging so as not to lose these economies of scale. In addition, local banks will find it optimal to target the multimarket bank because of the large surplus that can be obtained from merging with it. As a consequence, the multimarket bank will typically manage to be involved in the equilibrium merger.

This paper points to possible policy implications for the current consolidation process of the SBS. In practice, there presently exist limitations to the two optimal reactions that our paper unconvers: On the one hand, inter-regional mergers of savings banks may be de facto not allowed in some cases, due to regional politicians' motives. On the other hand, there are barriers for mergers between savings and commercial banks. Therefore, in a sense, this paper suggests the lift of these obstacles. At the time of this writing, a series of mergers backed by $€ 11.2$ billion in loans from the FROB (the Spanish government's bank rescue fund) has reduced the number of savings banks from 45 to 19 .

The rest of the paper is organized as follows. Section 2 presents the banking competition model. Sections 3 and 4 analyze the branching deregulation model and incentives to merge. Section 5 discusses some extensions of the model and Sect. 6 presents our conclusions. All the proofs are in the Appendix.

\section{Bank competition model}

Before describing the deregulation game and studying subsequent merger activities, we introduce the general banking competition framework in which we analyze the interplay between asymmetric geographic regulation and banking behavior. We need a model of spatial competition, and, following Economides et al. (1996) and Lozano-Vivas et al. (2010a), we rely on Salop's 1979 spatial model. We simplify matters by considering the limit case where transportation costs approach infinity. This way, we can dispense with interest rate decisions and focus on the number of branches established by each banking institution in each territory. Assuming the symmetric location of branches, profits to banking institution $i$ in territory $k$ amount to

$$
\pi_{i, k}=n_{i, k}\left(\delta_{k} \widetilde{r} \frac{1}{N_{k}}-\Phi\right)
$$

where $\delta_{k}$ represents territory $k$ 's deposit density, $\widetilde{r}$ is market interest rate, ${ }^{8} n_{i, k}$ is the number of branches opened by banking institution $i$ in territory $k, N_{k}$ is the total

\footnotetext{
8 We assume that banks, regardless of their territory, can access the same market interest rate.
} 
number of branches (of all banking institutions) in territory $k$, and $\Phi>0$ represents cost per branch. ${ }^{9}$

In this model, the cost to a bank depends positively on the absolute size of the branch network, but its returns depend on the relative size of the network. Hence, any additional branch always entails the same cost, although with decreasing marginal revenues. ${ }^{10}$

The profits to a banking institution that operates in several territories is the sum of its profits in each territory. Note that our formulation assumes that the functioning of one territory is isolated from that of the others (we do not allow customers to fulfill their banking needs outside their territory) and there are no economies of scale/scope in banking technology. ${ }^{11}$

\section{Asymmetric geographic regulation}

We consider a model with two territories and three players: two local banks and one multimarket bank. ${ }^{12}$ The two territories differ in deposit density, and, without loss of generality, we assume $\delta_{1}=\delta>0$ and $\delta_{2}=\alpha \delta$, with $\alpha \in(0,1]{ }^{13}$

Our starting point is the asymmetric regulation in which local banks are restricted to operate in their own territory whereas the multimarket bank is allowed to operate everywhere. Deregulation takes place as follows.

Definition 1 Once the regulatory agency announces the allowance of cross-border activities at a (future) given date,

Stage 1. Incumbents simultaneously decide on the number of branches to open in their own territories;

Stage 2. Upon observing incumbents' decisions in Stage 1 (once cross-border activities are allowed), the entrant decides how many branches to open in the new territory. Payoffs are given by the model described in Sect. 2.

\subsection{Initial conditions}

The initial conditions correspond to the situation in which local banks are restricted to operate in their own territory whereas the multimarket bank is allowed to operate everywhere. Let us denote each local bank by the territory in which it is initially

\footnotetext{
${ }^{9}$ In the main text we interpret $\Phi$ as the cost of opening branches, although it could also accommodate operative costs.

10 This bank competition model is strategically equivalent to a particular case of the rent-seeking game proposed by Tullock (1980).

11 Economies of scale associated with merger activities are allowed in Sect. 4, given that one of the main economic rationales for bank mergers is scale economies (see, e.g., Piloff and Santomero 1998, and Berger et al. 1999).

12 Section 5 discusses the implications of changing the number of players.

13 The parameter $\alpha$ measures how wealth (which will be deposited in the banking system) is distributed throughout the country. When $\alpha=1$, wealth is evenly distributed, whereas as $\alpha \rightarrow 0$ the percentage of wealth in territory 1 approaches $100 \%$. The Gini index of wealth is $\frac{1}{2} \frac{1-\alpha}{1+\alpha}$.
} 
Table 1 Equilibrium outcome in the regulated period

\begin{tabular}{llllll}
\hline Player & \multicolumn{2}{l}{ Market } & & & Branch network size \\
\cline { 2 - 3 } \cline { 5 - 6 } & 1 & 2 & & Absolute & Relative \\
\hline 1 & $8 \omega$ & 0 & & $8 \omega$ & $\frac{1}{2(1+\alpha)}$ \\
2 & 0 & $8 \alpha \omega$ & & $8 \alpha \omega$ & $\frac{\alpha}{2(1+\alpha)}$ \\
3 & $8 \omega$ & $8 \alpha \omega$ & & $8(1+\alpha) \omega$ & $1 / 2$ \\
Total & & & & $16(1+\alpha) \omega$ & 1 \\
\hline
\end{tabular}

constrained to operate, that is, 1 and 2. The subscript 3 is for the multimarket bank. The asymmetric regulatory body precluded local banks from operating outside their own territory. Hence, $n_{1,2}=n_{2,1}=0$. The multimarket bank is free to operate nationwide. We look for the Nash equilibrium $\left\{n_{k, k}^{*}, n_{3, k}^{*}\right\}$ of the banking competition game in every territory $k$.

Proposition 1 In territory $k$, the multimarket and local banks equally share the deposits market; each one opens $\frac{\delta_{k} \widetilde{r}}{4 \Phi}$ branches.

In equilibrium, the marginal revenue of branches equals the cost $\Phi$, and therefore the optimal number of branches must be decreasing in $\Phi$ and increasing in the revenue parameters $\delta_{k} \widetilde{r}$. The total number of branches in a given territory is $N_{k}=\frac{1}{2} \frac{\delta_{k} \widetilde{r}}{\Phi}$.

Let

$$
\omega=\frac{\delta \widetilde{r}}{32 \Phi}
$$

Table 1 displays the equilibrium outcome in this period.

As we see, each territory is equally shared by the multimarket bank and the local bank. This is the advantage conferred by asymmetric regulation to the multimarket banking sector: Its market share (1/2) exceeds its market representation $(1 / 3)$.

Given that territories differ in deposit densities, we obtain an asymmetric distribution of branch network sizes at the individual level: the multimarket bank enjoys the largest branch network, $8(1+\alpha) \omega$; the local bank settled in the richest territory is second, with $8 \omega$; and finally we have the other local bank, with $8 \alpha \omega$.

\subsection{The deregulation game}

We model the deregulation game as a standard incumbent-entrant model commonly used in the industrial organization literature. The main difference is that asymmetric regulation made the multimarket bank incumbent in both territories, whereas each local bank is an incumbent in one territory and an entrant in the other.

Accordingly, we assume that the regulatory authority announces that branching restrictions will be lifted. Recalling Definition 1 (Stages 1 and 2), we define a sequential game as follows. First, the incumbents of each territory can (prior to the date on which deregulation becomes effective) modify the number of their branches. Then, 
in the second stage, the entrants decide how many branches to open in the new territory. We look for the subgame perfect equilibrium of this game, starting from the last subgame and going backward.

\subsubsection{The entrant stage}

In this subgame, after observing the additional number of branches $I_{k}$ opened by the incumbents in territory $k$, entrants decide on the number of branches $e_{k}\left(I_{k}\right)$ to open.

Lemma 1 In the entry stage, the optimal number of branches opened by each entrant is

$$
e_{k}^{*}\left(I_{k}\right)= \begin{cases}\sqrt{2 N_{k}\left(I_{k}+N_{k}\right)}-\left(N_{k}+I_{k}\right) & \text { if } I_{k} \leq N_{k} \\ 0 & \text { otherwise }\end{cases}
$$

As we see, the optimal behavior of the entrant depends negatively on the branch network held by the incumbents. Hence, entry prevention is feasible, and achieved if the incumbents' network is doubled.

\subsubsection{The incumbents' stage}

We now analyze the behavior of the two incumbents. The incumbents decide on the optimal adjustment of their own network, anticipating the optimal behavior of the entrant described in Lemma 1. This optimal behavior is described in the next proposition.

Proposition 2 The optimal number of additional branches opened by each incumbent of territory $k$ is $N_{k} / 16$.

In other words, each incumbent finds it optimal to expand its branch network only by a factor of $1 / 32$. This expansion is not large enough to deter entry, since, as already pointed out, entry prevention requires the incumbents to double their network size. As a result, the entrant local bank opens $e_{k}^{*}\left(N_{k} / 8\right)=6 N_{k} / 16$ branches.

Even though the expansion of the entrant with respect to the expansion of the incumbents is large in relative terms, incumbents still hold a dominant position in the territory due to the large number of branches they had already installed during the regulated period.

\subsubsection{Equilibrium outcome of the deregulation game}

We can now set forth the equilibrium outcome of the deregulation game. The number of branches of each banking institution across territories is displayed in Table 2.

Note that in terms of relative size,

$$
\frac{9}{24}>\frac{9+6 \alpha}{24(1+\alpha)} \geq \frac{6+9 \alpha}{24(1+\alpha)}
$$


Table 2 Equilibrium outcome of the deregulation game (branch networks)

\begin{tabular}{|c|c|c|c|c|c|c|c|}
\hline \multirow[t]{3}{*}{ Player } & \multicolumn{7}{|c|}{ Absolute size } \\
\hline & \multicolumn{3}{|c|}{ Market 1} & \multicolumn{3}{|c|}{ Market 2} & \multirow[t]{2}{*}{ Aggregate } \\
\hline & Reg & Dereg & Total & Reg & Dereg & Total & \\
\hline 1 & $8 \omega$ & $\omega$ & $9 \omega$ & 0 & $6 \alpha \omega$ & $6 \alpha \omega$ & $(9+6 \alpha) \omega$ \\
\hline 2 & 0 & $6 \omega$ & $6 \omega$ & $8 \alpha \omega$ & $\alpha \omega$ & $9 \alpha \omega$ & $(6+9 \alpha) \omega$ \\
\hline 3 & $8 \omega$ & $\omega$ & $9 \omega$ & $8 \alpha \omega$ & $\alpha \omega$ & $9 \alpha \omega$ & $9(1+\alpha) \omega$ \\
\hline Total & & & $24 \omega$ & & & $24 \alpha \omega$ & $24(1+\alpha) \omega$ \\
\hline
\end{tabular}

which implies that at the individual level, the ranking of institutions by network size is 3,1 , and 2; that is, the multimarket bank enjoys the largest branch network $(9 / 24)$, the local bank originally settled in territory 1 is second (it also scores $9 / 24$ in the limit as $\alpha \rightarrow 0$ ), and the other local bank is last (there is a tie in case there are no asymmetries, i.e., $\alpha=1$ ). Note that the relative network size of the multimarket bank is $9 / 24$, irrespective of the relative strengths of the two territories. At the sector level, however, the local banks' sector enjoys a larger branch network $(15 / 24)$ than the multimarket's banking sector.

\section{Mergers and acquisitions}

As anticipated in the Introduction, we extend the deregulation game by considering the possibility of merger activities. We now contemplate this possibility in the post-deregulation era. ${ }^{14}$ We consider a game with two stages. First, there is a merger and acquisitions stage (M\&A stage hereafter) with two possible outcomes: a merger (involving two institutions) or no merger at all. ${ }^{15}$ If there is no merger, then the branch network remains unchanged and the payoff to each banking institution in a territory is simply the proportion of the total territory gains $\left(\delta_{k} \widetilde{r}\right)$ given by the relative size of the bank's branch network. This is so because branches were already installed in the deregulation game. The payoffs in this case are as follows:

$$
\begin{aligned}
& \bar{\pi}_{1}=\frac{9 \omega}{24 \omega} \delta_{1} \tilde{r}+\frac{6 \alpha \omega}{24 \alpha \omega} \delta_{2} \widetilde{r}=(12+8 \alpha) \widehat{\omega} \\
& \bar{\pi}_{2}=\frac{6 \omega}{24 \omega} \delta_{1} \widetilde{r}+\frac{9 \alpha \omega}{24 \alpha \omega} \delta_{2} \widetilde{r}=(8+12 \alpha) \widehat{\omega} \\
& \bar{\pi}_{3}=\frac{9 \omega}{24 \omega} \delta_{1} \tilde{r}+\frac{9 \alpha \omega}{24 \alpha \omega} \delta_{2} \widetilde{r}=(12+12 \alpha) \widehat{\omega}
\end{aligned}
$$

where

$$
\widehat{\omega}=\Phi \omega .
$$

\footnotetext{
14 The initial conditions of the M\&A game are not exogenous, but the equilibrium outcome of the deregulated game. Thus, we implicitly assume that in the deregulation game players do not anticipate future merging possibilities.

15 The model does not allow for full monopolization. This assumption is discussed in Sect. 5 .
} 
If a merger results from the M\&A stage, then there is a competition stage in which the (two) banking institutions decide on the number of their branches in the two territories. Given that one of the economic rationales for bank mergers is the presence of scale economies, we incorporate the possibility of economies of scale associated with merger activities in our model. ${ }^{16}$ To this aim, we assume that if two institutions, say $i$ and $j$, merge, the cost per branch for the merged institution is $\hat{\Phi}_{i+j} \leq \Phi .^{17}$

Let $\gamma_{i+j}=\Phi / \hat{\Phi}_{i+j}$. Note that, by assumption, $\gamma_{i+j} \geq 1$ and the higher $\gamma_{i+j}$, the higher the economies of scale associated with the merger $i+j$ with respect to remaining isolated. We assume that the cost reduction depends on the total size or profitability of the entities that were to merge, so bigger (more profitable) institutions enjoy larger cost reductions. In particular, we normalize $\gamma_{1+2}=1$ and consider $\gamma_{1+3}=\bar{\gamma} \geq 1$ and $\gamma_{2+3}=\rho \bar{\gamma}$, with $\rho \in\left[\frac{1}{\bar{\gamma}}, 1\right] .{ }^{18}$ In words, we consider that (i) a merger between the two smallest banks does not provide a cost reduction with respect to remaining isolated, ${ }^{19}$ (ii) a merger between the two largest banks provides the highest economies of scale (represented by $\bar{\gamma}$ ), and (iii) the economies of scale generated by the "intermediate" merger (between the smallest bank and the largest one) are a fraction $\rho$ of $\bar{\gamma}$. Clearly, if $\rho=\bar{\gamma}=1$, we get the situation in which there are no economies of scale.

We next solve for the subgame perfect equilibrium of the M\&A game, starting out from the competition stage.

\subsection{The competition stage}

The merged institution, say $i+j$, decides how many branches to operate in each territory at a unitary cost $\hat{\Phi}_{i+j}$. Afterward, the competitor (i.e., the bank that remains isolated) reacts and decides on its optimal number of branches at a unitary cost $\Phi$. The next proposition characterizes the equilibrium outcome of the competition stage.

Proposition 3 In each territory $k$, the final numbers of branches of the merged institution and the competitor are, respectively, $\frac{\delta_{k} \widetilde{r}}{4 \Phi}\left(\gamma_{i+j}\right)^{2}$ and $\frac{\delta_{k} \widetilde{r}}{4 \Phi}\left(2-\gamma_{i+j}\right) \gamma_{i+j}$.

Notice that the absolute size (i.e., network) of the merged institution is increasing in the parameter of the economies of scale $\left(\gamma_{i+j}\right)$, while that of the competitor is decreasing. This is intuitive, since economies of scale are associated with merger activities. If $\gamma_{i+j}=1$, then the merged institution and competitor would share each territory on an equal basis, reproducing the duopoly conditions of the regulated period.

\footnotetext{
16 See Dermine (1999) for an excellent analysis of the economies of bank mergers.

17 By definition, economies of scale mean lower costs for a given output. At this stage, however, interpretation of the branch cost as an opening cost or, alternatively, an operative cost becomes relevant. We want to adhere to the opening cost interpretation (as we did in the previous section), and therefore we assume that after merging, economies of scale materialize as revenue to the bank, rather than as a cost saving (as the operative cost interpretation requires). This revenue is materialized following this thought experiment: The merged bank sells its network at a unitary price $\Phi$ and then rebuilds it at a unitary price $\widehat{\Phi} \leq \Phi$.

18 We assume $\rho \geq \frac{1}{\bar{\gamma}}$ so that $\gamma_{2+3} \geq 1$.

19 This normalization does not affect substantially the results. As a consequence, $\bar{\gamma}$ and $\rho \bar{\gamma}$ are to be understood as economies of scale in excess to the ones produced by the merger between the two local banks.
} 
Table 3 Profits for every post-merger market structure

\begin{tabular}{lll}
\hline Market structure & Profits & \\
\cline { 2 - 3 } & Merged & Competitor \\
\hline $1+2,3$ & $23(1+\alpha) \widehat{\omega}$ & $17(1+\alpha) \widehat{\omega}$ \\
$1+3,2$ & $(15+8 \bar{\gamma})(1+\alpha) \widehat{\omega}+3 \widehat{\omega}$ & $\left(41-32 \bar{\gamma}+8 \bar{\gamma}^{2}\right)(1+\alpha) \widehat{\omega}-3 \widehat{\omega}$ \\
$2+3,1$ & $(15+8 \rho \bar{\gamma})(1+\alpha) \widehat{\omega}+3 \alpha \widehat{\omega}$ & $\left(41-32 \rho \bar{\gamma}+8 \rho^{2} \bar{\gamma}^{2}\right)(1+\alpha) \widehat{\omega}-3 \alpha \widehat{\omega}$ \\
\hline
\end{tabular}

Table 4 Surpluses for every post-merger market structure

\begin{tabular}{lll}
\hline Market structure & Surplus & \\
\cline { 2 - 3 } & Merged $\left(s_{i+j}\right)$ & Competitor $\left(s_{c}\right)$ \\
\hline $1+2,3$ & $3(1+\alpha) \widehat{\omega}$ & $5(1+\alpha) \widehat{\omega}$ \\
$1+3,2$ & $(8 \bar{\gamma}-5)(1+\alpha) \widehat{\omega}-\widehat{\omega}$ & $\left(29-32 \bar{\gamma}+8 \bar{\gamma}^{2}\right)(1+\alpha) \widehat{\omega}+\widehat{\omega}$ \\
$2+3,1$ & $(8 \rho \bar{\gamma}-5)(1+\alpha) \widehat{\omega}-\alpha \widehat{\omega}$ & $\left(29-32 \rho \bar{\gamma}+8 \rho^{2} \bar{\gamma}^{2}\right)(1+\alpha) \widehat{\omega}+\alpha \widehat{\omega}$ \\
\hline
\end{tabular}

The relative size (i.e., market share) of the merged institution is actually $\gamma_{i+j} / 2$, which implies that there is a point in the cost reduction $\left(\gamma_{i+j}=2\right)$ at which the merged institution expels the competitor from the market (at this point, the competitor finds it optimal to leave the market). In what follows, we assume $\bar{\gamma} \in[1,2]$.

Table 3 shows the bank profits for every post-merger market structure (see the Appendix for the required computations).

Given that the outside option -no merger- assigns participants their reservation values $\bar{\pi}$ given by (1), we need to compute the surplus to each bank, defined as profits net of reservation values. Thus, for each $\{i, j\} \in\{1,2,3\}$ and $c \in\{1,2,3\} \backslash\{i, j\}$, we denote by $s_{i+j}=\pi_{i+j}-\left(\bar{\pi}_{i}+\bar{\pi}_{j}\right)$ the surplus of the merger $i+j$, and by $s_{c}=\pi_{c}-\bar{\pi}_{c}$ the competitor surplus when the merger $i+j$ occurs. ${ }^{20}$ These surpluses are shown in Table 4.

The following are some useful observations.

(i) If the economies of scale associated with the merger $i+j$ are small (low $\gamma_{i+j}$ ), such a merger generates a positive surplus for both the merging institution and the competitor. Note also that in such a case the competitor surplus is larger than the merged institution surplus.

(ii) As the economies of scale associated with the merger $i+j$ become more important (high $\gamma_{i+j}$ ), the higher the surplus of the merging institution and the lower the surplus of the competitor (it is even negative for large values of $\gamma_{i+j}$ ).

With this information, we now step back and analyze the M\&A stage.

\footnotetext{
20 Note that $\pi_{i+j}$ and $\pi_{c}$ are the post-merger profits of the merged institution and the competitor, explicit in Table 3 , and $\bar{\pi}_{i}, \bar{\pi}_{j}$, and $\bar{\pi}_{c}$ are the banks' profits if no merger occurs, given by (1).
} 


\subsection{The M\&A stage}

This is the stage when M\&A are decided. To properly define the subgame to be solved at this stage, in addition to the equilibrium outcome of the competition stage, we need to explicitly define a merger protocol, that is, a description of the rules that determine when, by whom, over whom, and under which conditions a merger is proposed. First, we describe the surplus sharing rule (which implicitly determines the price that the acquiring bank pays to the acquiree) and then we state the protocol.

As regards the surplus sharing rule, we assume that the surplus from a merger is divided according to the profitability of the two merging institutions. ${ }^{21}$ Let $p_{i+j}^{i}=$ $\bar{\pi}_{i} /\left(\bar{\pi}_{i}+\bar{\pi}_{j}\right)$ be the share of the surplus of bank $i$ in the merger $i+j$, where $\bar{\pi}_{i}, \bar{\pi}_{j}$ are given by (1). Hence,

$$
\begin{array}{ll}
p_{1+2}^{1}=\frac{12+8 \alpha}{20+20 \alpha}, & p_{1+3}^{1}=\frac{12+8 \alpha}{24+20 \alpha} \\
p_{1+2}^{2}=\frac{8+12 \alpha}{20+20 \alpha}, & p_{2+3}^{2}=\frac{8+12 \alpha}{20+24 \alpha} \\
p_{1+3}^{3}=\frac{12+12 \alpha}{24+20 \alpha}, & p_{2+3}^{3}=\frac{12+12 \alpha}{20+24 \alpha}
\end{array}
$$

Then, the payoff that $i$ obtains from merging with $j$ is $\bar{\pi}_{i}+p_{i+j}^{i} s_{i+j}$. Straightforward calculations show that $\frac{1}{2}<p_{3+1}^{3}<p_{3+2}^{3}, p_{1+3}^{1}<\frac{1}{2}<p_{1+2}^{1}$ and $p_{2+3}^{2}<p_{2+1}^{2}<\frac{1}{2}$. Given these shares of the surplus, we can determine the target of each institution. The target of institution $i \in\{1,2,3\}$, denoted by $t_{i} \in\{1,2,3\} \backslash\{i\}$, is the bank that, across all institutions, provides $i$ with the highest payoff in the case of merging with $i$. Note that in the determination of the target of a given institution, an interesting tension arises between (i) the largest surplus that can be obtained from merging with a stronger rival and (ii) the higher share of the surplus that can be obtained by merging with a weaker rival.

Lemma 2 The target of each institutions is determined as follows:

(I) If $\bar{\gamma}<\frac{6+5 \alpha}{5+5 \alpha}, t_{1}=2$ whereas, if $\bar{\gamma}>\frac{6+5 \alpha}{5+5 \alpha}, t_{1}=3$.

(II) If $\rho \bar{\gamma}<\frac{5+6 \alpha}{5+5 \alpha}, t_{2}=1$ whereas, if $\rho \bar{\gamma}>\frac{5+6 \alpha}{5+5 \alpha}, t_{2}=3$.

(III) If $\rho<\frac{5+6 \alpha}{6+5 \alpha}, t_{3}=1$ whereas, if $\rho>\frac{5+6 \alpha}{6+5 \alpha}, t_{3}=2$.

The intuition is simple, if $\bar{\gamma}$ (resp. $\rho \bar{\gamma}$ ) is low, local bank 1 (resp. 2) prefers to merge with the other local bank, because, in case of merging with the multimarket bank, the latter gets the highest share of the surplus. In contrast, if $\bar{\gamma}$ (resp. $\rho \bar{\gamma}$ ) is large enough, the high surplus generated when merging to the multimarket bank compensates the low share of the local bank and, therefore, the multimarket bank becomes the target. On the other hand, if $\rho$ is large, the multimarket bank prefers to merge to the smallest local bank since it gets the highest surplus share without a significant loss in terms of

\footnotetext{
$\overline{21}$ We thank an anonymous referee for this suggestion. This natural formulation avoids introducing an ad hoc sequential mechanism of merger proposals and responses in which the respondent has lower bargaining power than the proposer (this was assumed in our former WP, Lozano-Vivas et al. 2010b).
} 
economies of scale. In contrast, if $\rho$ is low the surplus reduction does no longer make attractive for the multimarket bank to merge the smallest local bank.

Regarding the protocol, we consider that each bank can either initiate a merger process with its target or remain silent. If no merger process is initiated, then no merger occurs and the payoff to each institution is determined by (1). If just one merger process is initiated, then the corresponding merger takes place (note that all the mergers have a positive surplus and, therefore, the payoff to each of the merging institutions exceeds its reservation payoff). If two or more merger processes are initiated, there are two possibilities: (i) If two of the proposals coincide (i.e., two of the proposing banks are targets of each other), then the corresponding merger takes place, and (ii) otherwise, one of the initiated merger processes, randomly selected, succeeds. ${ }^{22}$

Since all mergers provide a positive surplus, in equilibrium there must be a merger (otherwise, any bank would have incentives to propose a merger with its target). In determining which institutions will be involved in the equilibrium merger, next lemma is very useful, as it shows that there is always a bank that is the common target of the other two institutions. This common target will necessarily be involved in the equilibrium merger and, therefore it will find it optimal to propose a merger with its target.

Lemma 3 For each $\alpha \in(0,1), \bar{\gamma} \in[1,2]$ and $\rho \in[1 / \bar{\gamma}, 1]$, there is $j \in\{1,2,3\}$ such that, for each $i \in\{1,2,3\} \backslash\{j\}, t_{i}=j$.

The following proposition shows the equilibrium outcome of the merger game.

Proposition 4 The equilibrium outcome of the merger game is as follows.

(I) If $\bar{\gamma}<\frac{5+6 \alpha}{5+5 \alpha}$, the two local banks merge with each other.

(II) If $\frac{5+6 \alpha}{5+5 \alpha}<\bar{\gamma}<\frac{6+5 \alpha}{5+5 \alpha}$, there are two possibilities: (i) if $\rho \bar{\gamma}<\frac{5+6 \alpha}{5+5 \alpha}$, the two local banks merge with each other; and (ii) if $\rho \bar{\gamma}>\frac{5+6 \alpha}{5+5 \alpha}$, the multimarket bank merges with a local bank.

(III) If $\frac{6+5 \alpha}{5+5 \alpha}<\bar{\gamma}<2-\sqrt{\frac{2+3 \alpha}{5+5 \alpha}}$, the multimarket bank merges with a local bank.

(IV) If $2-\sqrt{\frac{2+3 \alpha}{5+5 \alpha}}<\bar{\gamma}<\frac{6+5 \alpha}{3+3 \alpha}$, there are two possibilities: (i) if $\rho \bar{\gamma}>\frac{5+6 \alpha}{5+5 \alpha}$, the multimarket bank merges with a local bank; and (ii) if $\rho \bar{\gamma}<\frac{5+6 \alpha}{5+5 \alpha}$, either the multimarket bank merges with a local bank or the two local banks merge with each other, both outcomes being equally likely.

(V) If $\bar{\gamma}>\frac{6+5 \alpha}{3+3 \alpha}$, the multimarket bank merges with a local bank.

Figure 1 presents a graphical illustration of Proposition 4.

This proposition reveals that the multimarket bank enjoys an advantageous position in the merger game in the sense that the equilibrium outcome is typically her most preferred outcome. Consider first the case in which the two local banks merge with each other. This happens for low economies of scale $(\bar{\gamma})$. In this range, the driving force in the determination of the targets is the banks branch size. In this respect, the multimarket bank, which happens to be the largest one, is not targeted by any bank. As

\footnotetext{
22 Section 5 discusses the robustness of our results to other merger protocols used in the literature.
} 


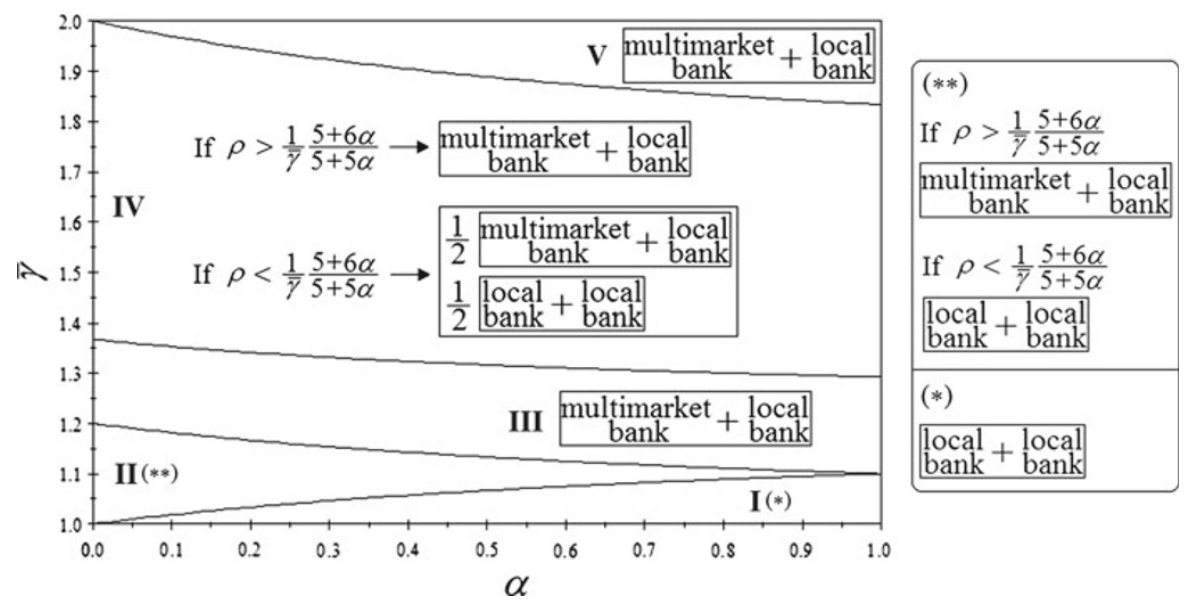

Fig. 1 Equilibrium outcome of the merger game

a result, the two local banks target to each other and, therefore, they merge with each other. In fact, this is the most preferred outcome for the multimarket bank because for low values of $\bar{\gamma}$ the merging activities have a public good nature: Banking institutions prefer a merger to take place (rather than no merger at all), but their most preferred situation is when their two competitors merge with each other.

Consider now the case in which the multimarket bank gets involved in the equilibrium merger. This happens for high enough economies of scale $(\bar{\gamma})$. In this case, the large surplus generated by a merger with the multimarket bank overweights the effect of the branch size in the determination of the targets. As a result, at least one local bank targets the multimarket bank. And it is precisely in this case in which the multimarket bank does not want to get stuck in the competitor's role because merging activities no longer have a public good nature.

This basic intuition is actually modulated by the parameter $\rho$, which measures the surplus generated by the "intermediate" merger $(2+3)$. Large values of $\rho$ imply that the multimarket bank and the small local bank target to each other. This has a direct effect on region II of Proposition 4 (low $\bar{\gamma}$ ), where the largest local bank is favoured by not being involved in the equilibrium merger. On the other hand, low values of $\rho$ makes the largest local bank to be the common target of the other two institutions. This has a direct effect in region IV of Proposition 4 (large $\bar{\gamma}$ ), where the merger activities have the public good nature for the multimarket bank but not for the small local bank. This is why the multimarket bank does not find it optimal to reciprocate the merger proposal by the large local bank, which opens the possibility of a merger between the two local banks with positive probability.

\section{Extensions}

This section analyzes four natural extensions of the basic model: (i) an increase in the number of banks and/or territories, (ii) allowance of full monopolization, 
(iii) the robustness of our results to changes in the merger protocol and (iv) changes in the timing.

\section{Changing the number of players}

Our banking competition model considers only two territories, two local banks and one multimarket bank. The main result is that the largest bank enjoys an advantageous position in the merger game in the sense that the equilibrium outcome is typically her most preferred outcome. Given that the asymmetric regulation gives an advantage to incumbents in terms of size, it easily follows that multimarket banks, which are incumbents in more territories than local banks, are at the top of the ranking in terms of size, assets, and so forth.

This advantage in terms of size is a distinguishing feature of the asymmetric regulation, and is therefore robust to an increase in the number of players. For example, if we enlarge the model by adding a second multimarket bank, in equilibrium there is no entry prevention, entrants expand on new territories, and the ranking of banking institutions by network size is not altered. The two multimarket banks become the largest banks, each of them with a branch network of $(5+5 \alpha) \frac{40}{27} \omega$. The local bank originally from the richest territory comes in third place, with a branch network of $(5+3 \alpha) \frac{40}{27} \omega$, and the remaining local bank enjoys the smallest branch network $(3+5 \alpha) \frac{40}{27} \omega$.

\section{The case of full monopolization}

The basic model only allows one merger, and therefore full monopolization as the result of a merger wave is not considered. However, there are incentives to reach full monopolization that stem from the assumption of infinite transportation costs. The reason is that a monopoly would find it optimal to have the smallest branch network admissible (recall that the strategy set is continuous) and hence, in the limit, its profits would be $\pi^{M}=(1+\alpha) \delta \widetilde{r}$; that is, the monopoly obtains the whole pie at negligible cost. In a duopoly, the industry profits comprise the whole pie $\left(\pi^{M}\right)$ net of branch network costs (which, in this case, are not negligible), making the incentive to reach the monopoly salient.

Even if only one merger is allowed, full monopolization is obtained in equilibrium if the economies of scale are large enough. In particular, a cost reduction of at least $50 \%\left(\gamma_{i+j} \geq 2\right)$ leads to a monopoly (cf. Proposition 3 ) because the bank who is left standing alone finds it optimal to leave the market.

\section{Robustness to different merger protocols}

In the model, we considered a merger game in which banking institutions simultaneously step ahead to initiate a merger. The resulting merger is selected among the proposals in a way that prioritizes common interests. ${ }^{23}$ In the literature, the proposal order is not usually decided by the players themselves, but results from the outset. There are two strands, random versus fixed, and two leading examples are Qiu and Zhou (2007) and Gorton et al. (2009), respectively.

\footnotetext{
23 There is actually only one arbitrariness: in the absence of common interest among the banks involved, the mechanism randomly selects one proposal.
} 
We now briefly analyze the equilibrium outcomes of the merger game if we had chosen the order proposals assumed by these protocols. ${ }^{24}$ We will see that the advantageous position hold by the multimarket bank is so powerful that our main result -its most preferred merger typically arises as the main equilibrium outcome-extends to these two protocols.

In the merger protocol of Qiu and Zhou (2007), the proposal order is random. At the beginning of each round, a bank is randomly selected as the proposer. The proposer can either pass or offer a merger. If the proposer passes, a new bank is selected to act as merger proposer. If all active banks pass, the M\&A stage ends. If the proposer offers a merger to another bank (respondent), the latter can either accept or reject it. If the respondent accepts, then the merger takes place. ${ }^{25}$ If the respondent rejects the merger, the M\&A stage ends.

In our setting, consider first the case in which the parameters of the economies of scale are small. The public good nature of the merging activities makes the multimarket bank not to get involved in any merger unless the random order places it in the last place. In this case, it would propose merging with the small local bank. This behavior is, in fact, anticipated by the small local bank that prefers to propose to the other local bank. Given that if a responder rejects one proposal the M\&A stage ends without mergers, the large local bank will accept to merge with the small one.

As the economies of scales start to grow, the public good nature of merging activities will sequentially vanish, and therefore some banks want to be involved in a merger. If one of these banks is lucky enough to be the first one to propose (this event has positive probability), then it will propose to its target and this merger will take place in equilibrium, because in this protocol, if a responder rejects one proposal the M\&A stage ends without mergers.

However, this result hinges on the feature that a rejection stops the M\&A stage without further mergers. Lifting this restriction leads to the protocol of Gorton et al. (2009) where a fixed proposal order, starting from the largest firm and ending with the smallest, is assumed and mergers can be safely rejected without stopping the merger order. In addition, there is at most one acquisition per firm and firms cannot acquire firms larger than themselves.

In our setting, the protocol of Gorton et al. (2009) gives the multimarket bank the opportunity to propose first and if no merger comes out from this stage, in the next one the large local bank can only propose a merger with the small local bank. Hence, the multimarket bank can always guarantee not to be involved in any merger by simply passing in the first stage of the merger game. As a result, the multimarket bank will manage to stand alone whenever merger activities have a public good nature for it. Also, when the parameters of the economies of scale are large, it will prefer to merge, and this is achievable because, for these configurations, the large local bank targets the multimarket bank rather than the small local bank.

\footnotetext{
24 In these protocols, the acquisition price comes from different bargaining procedures. Qiu and Zhou (2007) assume a take-it-or-leave-it bargaining process. Gorton et al. (2009) assume a Nash bargaining solution. In the upcoming discussion, we stick to our surplus share.

25 When a merger takes place, the merger game proceeds to the next round.
} 


\section{Timing of the merger game}

We finally analyze whether the results of the merger game would be altered if we introduce a sequential structure that reproduces more finely the process that the SBS has experienced in the post-deregulation era (where, initially, mergers between local and multimarket banks are not allowed). In this line, we shall consider the merger game in two stages: first the two local banks have the possibility to merge with each other. If they merge the game finished and, otherwise, the game proceeds to a second stage that reproduces the original merger game analyzed in Sect. 4.

When $\bar{\gamma}$ is low and, therefore, merger activities have a public good nature, the introduction of this initial stage does not have any effect on the equilibrium outcome. This result follows from the following two situations:

(i) In those cases in which the equilibrium outcome of the merger game analyzed in Sect. 4 is the merger between the two local banks, the introduction of the initial stage is innocuous.

(ii) In those cases in which the equilibrium outcome is a merger between the multimarket bank and a local bank (for instance, in region II when $\rho$ is high) we observe that, on the one hand, the local bank that is involved in the merger does in fact merge with its target and, in the other hand, the other local bank, given the public good nature, achieves its best outcome. Hence, no merger between the local banks is expected in the initial stage.

Only if $\bar{\gamma}$ is large and, therefore, mergers do no longer have a public good nature, the introduction of this initial stage may have an effect on the equilibrium outcome. In this case, in which (in our original merger game) the multimarket bank typically merges with a local bank, the local bank that is stuck in the competitor's role obtains quite a poor outcome (its surplus even possibly being negative -see Table 4). In this case, when we introduce the initial stage, such a local bank is eager to merge with the other one. In this line, the bank in a weak position would (strategically) be willing to give up part of the surplus that (given it relative size) it would get from the (potential) merger with the other local bank, so as to incentive the latter to merge. Hence if we denote by $i \in\{1,2\}$, the local bank in the weak position and $j \in\{1,2\} \backslash\{i\}$, we obtain that if $s_{1+2}>p_{j+3}^{j} s_{j+3}+s_{i}$ there is room for a merger between the two local banks in the initial stage. To illustrate that such a situation is feasible, consider the limit case where $\bar{\gamma}=2$ and $\rho=\alpha=1$. In this case, $t_{1}=t_{2}=3$ and $t_{3}=2$. Moreover, in our original merger game, banks 2 and 3 merge with each other. Hence, $i=1$ and $j=2$. Relying on (2) and Table 4, we obtain that, if $\bar{\gamma}=2$ and $\rho=\alpha=1, s_{1+2}=6 \widehat{\omega}$ and $p_{2+3}^{2} s_{2+3}+s_{1}=\frac{105}{11} \widehat{\omega}-5 \widehat{\omega}$. Hence $s_{1+2}-\left(p_{2+3}^{2} s_{2+3}+s_{1}\right)=\frac{16}{11} \widehat{\omega}>0$.

\section{Discussion and conclusion}

The paper so far has presented a stylized model of banking competition which can be used to assess the incentives to merge as a result of a structural change. We finish this paper by showing that it captures quite nicely the aggregate behavior of SBS in the last 30 years, as shown by the data for the period 1975-2009 provided in Table 7. Commercial banks were the only multimarket banks until 1989, since savings banks (local 
Table 5 Equilibrium outcome of the model and data outcome of the Spanish case

\begin{tabular}{|c|c|c|c|c|c|c|c|c|}
\hline & \multicolumn{8}{|c|}{ Relative size } \\
\hline & \multicolumn{3}{|c|}{ Commercial banks } & \multicolumn{5}{|c|}{ Savings banks } \\
\hline & Reg & Dereg & Var & Reg & Dereg & Var & & \\
\hline \multirow[t]{2}{*}{ Model } & 0.5 & 0.375 & 0.125 & 0.5 & 0.625 & 0.875 & In & 0.143 \\
\hline & & & & & & & Out & 0.857 \\
\hline \multirow[t]{2}{*}{ Data } & 0.598 & 0.468 & 0.127 & 0.402 & 0.532 & 0.873 & In & 0.128 \\
\hline & & & & & & & Out & 0.871 \\
\hline
\end{tabular}

Source: Own elaboration from Anuario Estadístico de las Cajas de Ahorro (1975-2009) and Anuario Estadístico de la Banca en España (1975-2009)

Table 6 Ranking of the current top five banking institutions in Spain

Source: Actualidad Económica, "Las 5000 mayores empresas españolas,” Reports, 1989-2009

\begin{tabular}{lccccc}
\hline Banking group & \multicolumn{5}{l}{ Years } \\
\cline { 2 - 6 } & 1990 & 1995 & 2000 & 2005 & 2009 \\
\hline Santander & 6 & 1 & 1 & 1 & 1 \\
BBVA & 1 & 2 & 2 & 2 & 2 \\
La Caixa & 4 & 5 & 3 & 3 & 3 \\
Caja Madrid & 8 & 6 & 4 & 5 & 4 \\
Banco Popular & 9 & 7 & 6 & 4 & 5 \\
CAM & 24 & 13 & 10 & 11 & 8 \\
Bancaja & 23 & 12 & 8 & 9 & 9 \\
Branch share savings & 0.4482 & 0.4590 & 0.5435 & 0.6049 & 0.6197 \\
banks & & & & & \\
\hline
\end{tabular}

banks) were allowed to open branches nationwide only at the end of 1988 . Table 5 compares the average market share (relative branch network sizes) of commercial and savings banks with the model's theoretical predictions for the regulated and deregulated periods. As we see, predictions are nicely confirmed by the data, specially the branch network expansion of commercial and savings banks in and out. ${ }^{26}$

The data presented above refer to the aggregate behavior of savings and commercial banks and therefore do not take into account heterogeneity in individual behavior. To tackle this issue, it is interesting to focus on the top banking institutions in the SBS. Table 6 reports the ranking variations of the current top five Spanish banking institutions in the last 20 years, based on yearly reports from Actualidad Economica on the 5,000 largest Spanish firms in terms of size, profits, and assets.

Recall that our model predicts a particular ranking in terms of size, assets, and profits, with commercial banks at the top, followed by local banks from wealthier and then poorer territories. This prediction is confirmed, as two of the top banking institutions are commercial banks, Santander and BBVA, which hold the top position

\footnotetext{
26 In refers to branches within their own territory and Out in those of others'.
} 
Table 7 Branch expansion (1975-2009)
Source: Anuario Estadístico de las Cajas de Ahorro (1975-2009) and Anuario Estadístico de la Banca en España (1975-2009)

a In refers to branches within their own territory and Out in those of others'

\begin{tabular}{|c|c|c|c|c|}
\hline \multirow[t]{2}{*}{ Years } & \multirow{2}{*}{$\begin{array}{l}\text { Commercial } \\
\text { Total }\end{array}$} & \multicolumn{3}{|c|}{ Savings $^{\mathrm{a}}$} \\
\hline & & Total & In & Out \\
\hline 1975 & 7569 & 6365 & & \\
\hline 1976 & 9093 & 6818 & & \\
\hline 1977 & 10205 & 7206 & & \\
\hline 1978 & 11095 & 7502 & & \\
\hline 1979 & 12235 & 7807 & & \\
\hline 1980 & 13223 & 8228 & & \\
\hline 1981 & 14290 & 8872 & & \\
\hline 1982 & 15374 & 9574 & & \\
\hline 1983 & 16046 & 10081 & & \\
\hline 1984 & 16399 & 10477 & & \\
\hline 1985 & 16568 & 10848 & & \\
\hline 1986 & 16471 & 11306 & & \\
\hline 1987 & 16449 & 11711 & & \\
\hline 1988 & 16651 & 12308 & & \\
\hline 1989 & 16623 & 13142 & 11699 & 1443 \\
\hline 1990 & 16835 & 13675 & 12001 & 1674 \\
\hline 1991 & 17923 & 13933 & 12013 & 1920 \\
\hline 1992 & 18154 & 14123 & 12024 & 2099 \\
\hline 1993 & 17713 & 14264 & 11997 & 2267 \\
\hline 1994 & 17405 & 14714 & 12115 & 2599 \\
\hline 1995 & 17841 & 15137 & 12422 & 2715 \\
\hline 1996 & 17657 & 15872 & 12579 & 3293 \\
\hline 1997 & 17727 & 16645 & 12899 & 3746 \\
\hline 1998 & 17593 & 17596 & 13161 & 4435 \\
\hline 1999 & 16948 & 18348 & 13431 & 4917 \\
\hline 2000 & 16027 & 19082 & 13821 & 5261 \\
\hline 2001 & 14928 & 19840 & 13790 & 6050 \\
\hline 2002 & 14209 & 20347 & 14023 & 6324 \\
\hline 2003 & 14204 & 20891 & 14288 & 6603 \\
\hline 2004 & 14309 & 21527 & 14493 & 7034 \\
\hline 2005 & 14661 & 22443 & 14661 & 7782 \\
\hline 2006 & 15230 & 23455 & 14863 & 8592 \\
\hline 2007 & 15578 & 24635 & 15076 & 9559 \\
\hline 2008 & 15638 & 25033 & 15087 & 9946 \\
\hline 2009 & 14879 & 24250 & 14609 & 9641 \\
\hline
\end{tabular}

most years (although Santander started in sixth position in 1990). The ranking also shows that savings banks originally settled in the two wealthiest Spanish regions (Cataluña and Madrid) come next: La Caixa and Caja Madrid. Two additional saving 
banks, CAM and Bancaja, originally settled in Comunidad Valenciana (a slightly less wealthy region), come next.

The model's good fit with the recent evolution of the SBS encourages us to be confident about the applicability of merger predictions. A critical issue in this respect is the relevant region of the parameters space, for the optimal merger depends on the size of the economies of scale and the wealth distribution. We end the paper by calibrating the model.

As regards the wealth distribution, the Gini index in our model is $\frac{1}{2} \frac{1-\alpha}{1+\alpha}$. Since the Spanish Gini index is 0.313 (Eurostat, 2007 - Official statistics, European Commission), we obtain a calibrated value of $\alpha=0.23$. Also, the calibrated values for the bounds of the relevant regions of Proposition 4 are $\frac{5+6 \alpha}{5+5 \alpha}=1.037$ and $\frac{6+5 \alpha}{5+5 \alpha}=1.163$. As a result, we conclude that the optimal merger will involve the commercial bank whenever $\rho \bar{\gamma}>1.037$ or $\bar{\gamma}>1.163$; otherwise, the savings banks will merge with each other. $^{27}$

Indeed, it is worth mentioning that mergers are the natural consequence of the deregulation and that the two kind of mergers which our model yields as optimal are, to some extent, deterred in the current state of the SBS: in some cases inter-regional mergers between savings banks are avoided due to regional politicians' motives, and there exist barriers for mergers between savings and commercial banks.

Open Access This article is distributed under the terms of the Creative Commons Attribution License which permits any use, distribution and reproduction in any medium, provided the original author(s) and source are credited.

\section{Appendix}

Proof of Proposition 1 For each $i \in\{k, 3\}$

$$
n_{i, k}^{*} \in \arg \max _{\left\{n_{i, k}\right\}} n_{i, k}\left(\delta_{k} \widetilde{r} \frac{1}{n_{i, k}+n_{-i, k}^{*}}-\Phi\right)
$$

The first-order condition $\frac{\partial \pi_{i, k}}{\partial n_{i, k}}=0$ yields

$$
\frac{n_{-i, k}^{*}}{\left(n_{i, k}+n_{-i, k}^{*}\right)^{2}}=\frac{\Phi}{\delta_{k} \widetilde{r}}
$$

\footnotetext{
27 Proposition 4 shows that for the range $\rho \bar{\gamma}<1.037$ and $\bar{\gamma} \in[1.339,1.938]$ the merger between the two savings banks has also positive probability. Note however that the economies of scale associated to these values of $\bar{\gamma}$ are really large. This can be observed by computing the largest value of $\bar{\gamma}$ compatible with a reduction in the aggregate branch network of the merging institutions. It happens to be the value of $\bar{\gamma}$ that satisfies $8(1+\alpha) \bar{\gamma}^{2} /(18+15 \alpha)=1$ (cf. Table 2 and Proposition 3), which for the calibrated value of $\alpha$ is 1.47 .
} 
Imposing symmetry, we get

$$
n_{k, k}^{*}=n_{3, k}^{*}=\frac{\delta_{k}}{4} \frac{\widetilde{r}}{\Phi}
$$

as requested.

Proof of Lemma 1 Let $I_{k}$ be the total number of branches opened by the two incumbents in territory $k$, and $e_{k}$ the number of branches opened by the entrant. The problem of the entrant is

$$
e_{k}^{*} \in \arg \max _{\left\{e_{k}\right\}} e_{k}\left(\delta_{k} \widetilde{r} \frac{1}{e_{k}+I_{k}+N_{k}}-\Phi\right)
$$

The first-order condition yields

$$
2 N_{k}\left(I_{k}+N_{k}\right)=\left(e_{k}^{*}+I_{k}+N_{k}\right)^{2}
$$

Hence

$$
e_{k}^{*}=\sqrt{2 N_{k}\left(I_{k}+N_{k}\right)}-\left(N_{k}+I_{k}\right)
$$

It is easy to show that the optimal number of branches opened by each entrant is decreasing in $I_{k}$ and that it is null when the incumbent opens $N_{k}$ branches.

Proof of Proposition 2 Let $i_{k}$ and $\hat{\imath}_{k}$ be the number of new branches opened by each of the two incumbents of territory $k$. The problem of an incumbent is

$$
i_{k}^{*} \in \arg \max _{\left\{i_{k}\right\}} i_{k}\left(\delta_{k} \widetilde{r} \frac{1}{i_{k}+\hat{\imath}_{k}^{*}+N_{k}+e_{k}^{*}\left(i_{k}+\hat{\imath}_{k}^{*}\right)}-\Phi\right)
$$

The first-order condition yields

$$
\frac{\hat{\imath}_{k}^{*}+N_{k}+e_{k}^{*}\left(i_{k}+\hat{\imath}_{k}^{*}\right)-\frac{\partial e_{k}^{*}\left(i_{k}+\hat{\imath}_{k}^{*}\right)}{\partial i_{k}}}{\left(i_{k}+\hat{\imath}_{k}^{*}+N_{k}+e_{k}^{*}\left(i_{k}+\hat{\imath}_{k}^{*}\right)\right)^{2}}=\frac{1}{2 N_{k}}
$$

where

$$
\frac{\partial e_{k}^{*}\left(i_{k}+\hat{\imath}_{k}^{*}\right)}{\partial i_{k}}=\frac{e_{k}^{*}\left(i_{k}+\hat{\imath}_{k}^{*}\right)-\left(i_{k}+\hat{\imath}_{k}^{*}+N_{k}\right)}{2\left(i_{k}+\hat{\imath}_{k}^{*}+N_{k}\right)}
$$

Note that this derivative is linear in $e_{k}^{*}(\cdot)$. Imposing symmetry $\left(i_{k}^{*}=\hat{\imath}_{k}^{*}\right)$, we get

$$
i_{k}^{*}=\frac{N_{k}}{16}
$$


Proof of Proposition 3 Let $m_{k}$ be the number of branches that the merged institution has decided to have in territory $k$ once the merger takes place. Let $q_{k}$ be the number of (old) branches the competitor had in territory $k$ prior to the merger, and $a_{k}$ the number of additional branches of the competitor in territory $k$ after the merger (note that $a_{k}$ can be negative). The problem of the competitor in territory $k$ is

$$
a_{k}^{*} \in \arg \max _{\left\{a_{k}\right\}} \delta_{k} \tilde{r} \frac{q_{k}+a_{k}}{q_{k}+a_{k}+m_{k}}-a_{k} \Phi
$$

The first-order condition yields

$$
\delta_{k} \widetilde{r} \frac{m_{k}}{\left(q_{k}+a_{k}+m_{k}\right)^{2}}-\Phi=0
$$

Solving, we get

$$
a_{k}^{*}=\sqrt{\frac{\delta_{k} \widetilde{r}}{\Phi} m_{k}}-m_{k}-q_{k}
$$

Once the optimal reaction of the competitor has been computed, we step back to find the optimal number of branches of the merged institution in territory $k$. Given the asymmetry in the costs of old and new branches derived from the economies of scale associated with the merger (the new branches of the merged institution are less costly than the old ones), the optimal strategy implies first closing all its own existing branches in territory $k$ (recovering $\Phi$ per branch) and then opening $m_{k}^{*}$ new branches afresh (at a unitarian cost of $\hat{\Phi}_{i+j}<\Phi$ ), where $m_{k}^{*}$ results from

$$
m_{k}^{*} \in \arg \max _{\left\{m_{k}\right\}} \delta_{k} \widetilde{r} \frac{m_{k}}{m_{k}+q_{k}+a_{k}^{*}\left(m_{k}\right)}-m_{k} \hat{\Phi}_{i+j}
$$

The first-order condition yields

$$
\Phi \frac{\sqrt{\frac{\delta_{k} \widetilde{r}}{\Phi} m_{k}^{*}}}{2 m_{k}^{*}}-\hat{\Phi}_{i+j}=0
$$

Solving, we get

$$
m_{k}^{*}=\frac{\delta_{k} \widetilde{r}}{4 \Phi}\left(\frac{\Phi}{\hat{\Phi}_{i+j}}\right)^{2}=\frac{\delta_{k} \widetilde{r}}{4 \Phi} \gamma_{i+j}^{2}
$$

Thus

$$
q_{k}+a_{k}^{*}\left(m_{k}^{*}\right)=\sqrt{\frac{\delta_{k} \widetilde{r}}{\Phi} \frac{\delta_{k} \widetilde{r}}{4 \Phi} \gamma_{i+j}^{2}}-\frac{\delta_{k} \widetilde{r}}{4 \Phi} \gamma_{i+j}^{2}=\frac{\delta_{k} \widetilde{r}}{4 \Phi}\left(2-\gamma_{i+j}\right) \gamma_{i+j}
$$


Proof of Lemma 2 We first obtain the target of bank 1. If it merges with bank 2, its payoff is $\bar{\pi}_{1}+p_{1+2}^{1} s_{1+2}$, and if it merges with bank 3 , its payoff is $\bar{\pi}_{1}+p_{1+3}^{1} s_{1+3}$, where $p_{1+2}^{1} s_{1+2}=\frac{12+8 \alpha}{20+20 \alpha} 3(1+\alpha) \widehat{\omega}$ and $p_{1+3}^{1} s_{1+3}=\frac{12+8 \alpha}{24+20 \alpha}((8 \bar{\gamma}-5)(1+\alpha)-1) \widehat{\omega}$. Some algebra shows that $p_{1+2}^{1} s_{1+2}>p_{1+3}^{1} s_{1+3}$ if and only if $\bar{\gamma}<\frac{6+5 \alpha}{5+5 \alpha}$.

Regarding bank 2 , if it merges with bank 1 , its payoff is $\bar{\pi}_{2}+p_{1+2}^{2} s_{1+2}$, and if it merges with bank 3 , its payoff is $\bar{\pi}_{2}+p_{2+3}^{2} s_{2+3}$, where $p_{1+2}^{2} s_{1+2}=\frac{8+12 \alpha}{20+20 \alpha} 3(1+\alpha) \widehat{\omega}$ and $p_{2+3}^{2} s_{2+3}=\frac{8+12 \alpha}{20+24 \alpha}((8 \rho \gamma-5)(1+\alpha)-\alpha) \widehat{\omega}$. Some algebra shows that $p_{1+2}^{2} s_{1+2}>p_{2+3}^{2} s_{2+3}$ if and only if $\rho \bar{\gamma}<\frac{5+6 \alpha}{5+5 \alpha}$.

Finally, regarding bank 3 , if it merges with bank 1 , its payoff is $\bar{\pi}_{3}+p_{1+3}^{3} s_{1+3}$, and if it merges with bank 2 , it gets $\bar{\pi}_{3}+p_{2+3}^{3} s_{2+3}$, where $p_{1+3}^{3} s_{1+3}=$ $\frac{(12+12 \alpha)((8 \gamma-5)(1+\alpha)-1) \widehat{\omega}}{24+20 \alpha}$ and $p_{2+3}^{3} s_{2+3}=\frac{12+12 \alpha}{20+24 \alpha}((8 \rho \gamma-5)(1+\alpha)-\alpha) \widehat{\omega}$. Some algebra shows that $p_{1+3}^{3} s_{1+3}>p_{2+3}^{3} s_{2+3}$ if and only if $\rho<\frac{5+6 \alpha}{6+5 \alpha}$.

Proof of Lemma 3 Let $\bar{\gamma}<\frac{5+6 \alpha}{5+5 \alpha}$. Then $\bar{\gamma}<\frac{6+5 \alpha}{5+5 \alpha}$ and, since $\rho \leq 1, \rho \bar{\gamma}<\frac{5+6 \alpha}{5+5 \alpha}$. Hence, by Lemma 2, $t_{1}=2$ and $t_{2}=1$ and the result follows.

Now let $\frac{5+6 \alpha}{5+5 \alpha}<\bar{\gamma}<\frac{6+5 \alpha}{5+5 \alpha}$. By Lemma 2, $t_{1}=2$. If $\rho<\frac{5+6 \alpha}{6+5 \alpha}$, since $\rho \bar{\gamma}<$ $\frac{5+6 \alpha}{6+5 \alpha} \frac{6+5 \alpha}{5+5 \alpha}=\frac{5+6 \alpha}{5+5 \alpha}$, by Lemma $2, t_{2}=1$ and the result follows. If $\rho>\frac{5+6 \alpha}{6+5 \alpha}$, by Lemma $2 t_{3}=2$. Since $t_{1}=t_{3}=2$, no matter the value of $t_{2}$, the result follows

Finally, let $\bar{\gamma}>\frac{6+5 \alpha}{5+5 \alpha}$. Then, by Lemma $2, t_{1}=3$. There are two possibilities: If $\rho<\frac{5+6 \alpha}{6+5 \alpha}$ then, by Lemma $2, t_{3}=1$ and the result follows. If $\rho>\frac{5+6 \alpha}{6+5 \alpha}$ then $t_{3}=2$ but, since $\bar{\gamma}>\frac{6+5 \alpha}{5+5 \alpha}$ and $\rho>\frac{5+6 \alpha}{6+5 \alpha}$ imply $\rho \bar{\gamma}>\frac{5+6 \alpha}{5+5 \alpha}$, by Lemma $2, t_{2}=3$.

Proof of Proposition 4 We analyze separately two parts in this proof: part 1 $\left(\bar{\gamma}<\frac{6+5 \alpha}{5+5 \alpha}\right)$ and part $2\left(\bar{\gamma}>\frac{6+5 \alpha}{5+5 \alpha}\right)$.

Part 1) Let $\bar{\gamma}<\frac{6+5 \alpha}{5+5 \alpha}$. By Lemma $2, t_{1}=2$. There are three relevant cases:

(1.i) Let $\rho<\frac{5+6 \alpha}{6+5 \alpha}$. Then, by Lemma 2, $t_{3}=1$. Moreover $\rho \bar{\gamma}<\frac{5+6 \alpha}{6+5 \alpha} \frac{6+5 \alpha}{5+5 \alpha}=\frac{5+6 \alpha}{5+5 \alpha}$ and, by Lemma $2, t_{2}=1$. Since all mergers provide a positive surplus, in equilibrium, there must be a merger (otherwise, any bank would have incentives to propose a merger to its target). Thus, since local bank 1 is the common target of the other two institutions, it will be involved in the (equilibrium) merger for sure. Therefore, its optimal strategy is to propose a merger with its target (bank 2), which weakly increases the chances of its preferred merger. Then, given bank 1's optimal strategy, if bank 2 chooses to propose a merger to its target (bank 1), the merger takes place with certainty, and bank 2 's profits are $\bar{\pi}_{2}+p_{1+2}^{2} \cdot s_{1+2}$. If bank 2 decides to remain silent, its (expected) payoff, depending on bank 3's action (to propose a merger to bank 1 , or not), can be $\bar{\pi}_{2}+\frac{1}{2}\left(p_{1+2}^{2} \cdot s_{1+2}+s_{2}\right)$ or $\bar{\pi}_{1}+p_{1+2}^{2} \cdot s_{1+2}$ (note that the second case does not make a difference for bank 2 ). Hence, it is optimal for bank 2 to remain silent if $s_{2}>p_{1+2}^{2} \cdot s_{1+2}$. Using the expressions for $s_{1+2}$ and $s_{2}$ provided in Table 4 and $p_{1+2}^{2}$ given by (2), some algebra shows that $s_{2}>p_{1+2}^{2} \cdot s_{1+2}$ if and only if $\bar{\gamma}<2-\sqrt{\frac{2+3 \alpha}{5+5 \alpha}}$. Hence, since $\frac{6+5 \alpha}{5+5 \alpha}<2-\sqrt{\frac{2+3 \alpha}{5+5 \alpha}}$ for all $\alpha$, bank 2 remains silent. We finally analyze bank 3 's optimal response. If bank 3 also chooses to remain silent, there is a merger between the two local banks, and bank 3's payoff is $\bar{\pi}_{3}+s_{3}$. In contrast, if 
bank 3 decides to propose a merger (to bank 1), there would be two merger proposals (none of them reciprocated). In such a case, a merger occurs between either banks 1 and 2 or banks 3 and 1, both of them with equal probability, and bank 3's (expected) payoff is $\bar{\pi}_{3}+\frac{1}{2}\left(p_{1+3}^{3} \cdot s_{1+3}+s_{3}\right)$. Hence, it is optimal for bank 3 to remain silent if $s_{3}>p_{1+3}^{3} \cdot s_{1+3}$. Some algebra shows that $s_{3}>p_{1+3}^{3} \cdot s_{1+3}$ if and only if $\bar{\gamma}<\frac{6+5 \alpha}{3+3 \alpha}$. Since $\bar{\gamma}<\frac{6+5 \alpha}{5+5 \alpha}<\frac{6+5 \alpha}{3+3 \alpha}$, bank 3 remains silent. Thus, in case (1.i), the two local banks merge with each other in equilibrium.

(1.ii) Let $\rho>\frac{5+6 \alpha}{6+5 \alpha}$ and $\rho \bar{\gamma}<\frac{5+6 \alpha}{5+5 \alpha}$. Then, by Lemma $2, t_{3}=2$ and $t_{2}=1$. In this case it is local bank 2 (the common target) the institution that will be involved in the (equilibrium) merger for sure. Therefore, bank 2 proposes a merger with its target (bank 1). Analogously to the previous case, it is optimal for bank 1 to remain silent if $s_{1}>p_{1+2}^{1} \cdot s_{1+2}$. Some algebra shows that $s_{1}>p_{1+2}^{1} \cdot s_{1+2}$ if and only if $\rho \bar{\gamma}<2-\sqrt{\frac{3+2 \alpha}{5+5 \alpha}}$. Hence, since $\frac{5+6 \alpha}{5+5 \alpha}<2-\sqrt{\frac{3+2 \alpha}{5+5 \alpha}}$ for all $\alpha$, bank 1 remains silent. We finally analyze bank 3's optimal response. Analogously to the previous case, we conclude that it is optimal for bank 3 to remain silent if $s_{3}>p_{2+3}^{3} \cdot s_{2+3}$ and to propose a merger (to bank 1) otherwise. Some algebra shows that $s_{3}>p_{2+3}^{3} \cdot s_{2+3}$ if and only if $\rho \bar{\gamma}<\frac{5+6 \alpha}{3+3 \alpha}$. Since $\rho \bar{\gamma}<\frac{5+6 \alpha}{5+5 \alpha}<\frac{5+6 \alpha}{3+3 \alpha}$, bank 3 remains silent. Thus, also in case (1.ii), the two local banks merge with each other in equilibrium.

(1.iii) Let $\rho>\frac{5+6 \alpha}{6+5 \alpha}$ and $\rho \bar{\gamma}>\frac{5+6 \alpha}{5+5 \alpha}$. Then, by Lemma $2, t_{3}=2$ and $t_{2}=3$. In this case it is local bank 2 (the common target) the institution that will be involved in the (equilibrium) merger for sure. Therefore, bank 2 proposes a merger with its target (bank 3). Analogously to the previous cases, it is optimal for bank 3 to remain silent if $s_{3}>p_{2+3}^{3} \cdot s_{2+3}$. Some algebra shows that $s_{3}>p_{2+3}^{3} \cdot s_{2+3}$ if and only if $\rho \bar{\gamma}<\frac{5+6 \alpha}{3+3 \alpha}$. Hence, since $\rho \bar{\gamma}<\bar{\gamma}<\frac{6+5 \alpha}{5+5 \alpha}<\frac{5+6 \alpha}{3+3 \alpha}$ for all $\alpha$, bank 3 remains silent. We finally analyze bank 1's optimal response. Analogously to the previous case, we conclude that it is optimal for bank 1 to remain silent if $s_{1}>p_{1+2}^{1} \cdot s_{1+2}$ and to propose a merger (to bank 2) otherwise. Some algebra shows that $s_{1}>p_{1+2}^{1} \cdot s_{1+2}$ if and only if $\rho \bar{\gamma}<2-\sqrt{\frac{3+2 \alpha}{5+5 \alpha}}$. Since $\rho \bar{\gamma}<\bar{\gamma}<\frac{6+5 \alpha}{5+5 \alpha}<2-\sqrt{\frac{3+2 \alpha}{5+5 \alpha}}$ for all $\alpha$, bank 1 remains silent. Thus, in case (1.iii), bank 3 and bank 2 merge with each other in equilibrium.

We now summarize part 1: Assuming $\bar{\gamma}<\frac{6+5 \alpha}{5+5 \alpha}$, if $\rho \bar{\gamma}>\frac{5+6 \alpha}{5+5 \alpha}$ (case 1.iii) bank 3 and bank 2 merge with each other (note that $\bar{\gamma}<\frac{6+5 \alpha}{5+5 \alpha}$ and $\rho \bar{\gamma}>\frac{5+6 \alpha}{3+3 \alpha}$ imply $\rho>\frac{5+6 \alpha}{6+5 \alpha}$ ), and otherwise (cases 1.i and 1.ii), the two local banks merge with each other. Since $\rho \bar{\gamma}>\frac{5+6 \alpha}{5+5 \alpha}$ implies $\bar{\gamma}>\frac{5+6 \alpha}{5+5 \alpha}$, this proves (I)-(II).

Part 2) Let $\bar{\gamma}>\frac{6+5 \alpha}{5+5 \alpha}$. By Lemma $2, t_{1}=3$. There are two relevant cases:

(2.i) $\rho \bar{\gamma}>\frac{5+6 \alpha}{5+5 \alpha}$. By Lemma $2, t_{2}=3$. Since all mergers provide a positive surplus, in equilibrium, there must be a merger (otherwise, any bank would have incentives to propose a merger to its target). Thus, since the multimarket bank is the common target of the other two institutions, it will be involved in the (equilibrium) merger for sure.

(2.ii) $\rho \bar{\gamma}<\frac{5+6 \alpha}{5+5 \alpha}$. By Lemma $2, t_{2}=1$. Since $\bar{\gamma}>\frac{6+5 \alpha}{5+5 \alpha}$ and $\rho \bar{\gamma}<\frac{5+6 \alpha}{5+5 \alpha}$, we get, respectively, $\frac{1}{\bar{\gamma}}<\frac{5+5 \alpha}{6+5 \alpha}$ and $\rho<\frac{1}{\bar{\gamma}} \frac{5+6 \alpha}{5+5 \alpha}$. Hence $\rho<\frac{5+5 \alpha}{6+5 \alpha} \frac{5+6 \alpha}{5+5 \alpha}=\frac{5+6 \alpha}{6+5 \alpha}$ and, by Lemma $2, t_{3}=1$. Thus, in this case it is local bank 1 (the common target) the institution that will be involved in the (equilibrium) merger for sure. Therefore, bank 1 proposes 
a merger with its target (bank 3). Then, it is optimal for bank 3 to remain silent if $s_{3}>p_{1+3}^{3} \cdot s_{1+3}$ and to propose a merger to bank 1 otherwise. Some algebra shows that $s_{3}>p_{1+3}^{3} \cdot s_{1+3}$ if and only if $\bar{\gamma}<\frac{6+5 \alpha}{3+3 \alpha}$. Hence, if $\bar{\gamma}>\frac{6+5 \alpha}{3+3 \alpha}$ there is a merger between banks 1 and 3 . Assume now that $\bar{\gamma}<\frac{6+5 \alpha}{3+3 \alpha}$, then bank 3 remains silent. We finally analyze bank 2's optimal response. It is optimal for bank 2 to remain silent if $s_{2}>p_{1+2}^{2} \cdot s_{1+2}$ and to propose a merger (to bank 1) otherwise. Some algebra shows that $s_{2}>p_{1+2}^{2} \cdot s_{1+2}$ if and only if $\bar{\gamma}<2-\sqrt{\frac{2+3 \alpha}{5(1+\alpha)}}$. Hence, if $\bar{\gamma}<2-\sqrt{\frac{2+3 \alpha}{5(1+\alpha)}}$ bank 2 remains silent and, therefore, there is a merger between banks 1 and 3. On the other hand, if $\bar{\gamma}>2-\sqrt{\frac{2+3 \alpha}{5(1+\alpha)}}$, bank 2 proposes a merger to bank 1 and, therefore, there are two mergers proposals (from bank 1 to bank 3 and from bank 2 to bank 1), none of them reciprocated. Hence, each of the two proposals succeeds with a probability of $1 / 2$.

We now summarize part 2: Assuming $\bar{\gamma}>\frac{6+5 \alpha}{5+5 \alpha}$, from the analysis of cases $2 . i$ and 2.ii, we conclude that in equilibrium bank 3 merges with one of the local banks with a probability of one, except for the situation in which the following three conditions (jointly) hold: $\rho \bar{\gamma}<\frac{5+6 \alpha}{5+5 \alpha}, \bar{\gamma}<\frac{6+5 \alpha}{3+3 \alpha}$ and $\bar{\gamma}>2-\sqrt{\frac{2+3 \alpha}{5+5 \alpha}}$. In the latter case, either banks 1 and 3 or banks 1 and 2 merge with each other, both events being equally likely. This proves (III)-(V).

\section{Analysis of post-merger market structures.}

- $(1+2,3)$ : The two local banks merge with each other.

In this case, by Proposition 3 (see also Table 2), the merged institution closes $15(1+\alpha) \omega$ old branches (at $\Phi$ monetary units per branch), opens $\frac{\delta \widetilde{r}}{4 \Phi}(1+\alpha) \gamma_{1+2}^{2}$ new branches (at $\hat{\Phi}_{1+2}$ units per branch), and, in each territory, gets a share of $\gamma_{1+2} / 2$ of the market. Hence, its payoff is

$$
\begin{aligned}
\pi_{1+2} & =(1+\alpha) 15 \frac{\delta \widetilde{r}}{32 \Phi} \Phi-(1+\alpha) \gamma_{1+2}^{2} \frac{\delta \widetilde{r}}{4 \Phi} \hat{\Phi}_{1+2}+(1+\alpha) \frac{\gamma_{1+2}}{2} \delta \widetilde{r} \\
& =\left(15+8 \gamma_{1+2}\right)(1+\alpha) \widehat{\omega}=23(1+\alpha) \widehat{\omega}
\end{aligned}
$$

The multimarket bank closes $\left(9 \omega-\frac{\delta \widetilde{r}}{4 \Phi}\left(2-\gamma_{1+2}\right) \gamma_{1+2}\right)(1+\alpha)$ branches (at $\Phi$ units per branch) and, in each territory, gets a share $\left(2-\gamma_{1+2}\right) / 2$ of the market. Hence, its payoff is

$$
\begin{aligned}
\pi_{3} & =(1+\alpha)\left(9 \frac{\delta \widetilde{r}}{32 \Phi} \Phi-\left(2-\gamma_{1+2}\right) \gamma_{1+2} \frac{\delta \widetilde{r}}{4 \Phi} \Phi\right)+(1+\alpha) \frac{2-\gamma_{1+2}}{2} \delta \widetilde{r} \\
& =\left(41-32 \gamma_{1+2}+8 \gamma_{1+2}^{2}\right)(1+\alpha) \widehat{\omega}=17(1+\alpha) \widehat{\omega}
\end{aligned}
$$

- $(1+3,2):$ The multimarket bank and local bank 1 merge with each other.

The merged institution closes $(18+15 \alpha) \omega$ old branches (at $\Phi$ units per branch), opens $\frac{\delta \widetilde{r}}{4 \Phi}(1+\alpha) \gamma_{1+3}^{2}$ new branches (at $\hat{\Phi}_{1+3}$ units per branch), and, in each territory, 
gets a share $\gamma_{1+3} / 2$ of the market. Hence, its payoff is

$$
\begin{aligned}
\pi_{1+3} & =(18+15 \alpha) \frac{\delta \widetilde{r}}{32 \Phi} \Phi-(1+\alpha) \gamma_{1+3}^{2} \frac{\delta \widetilde{r}}{4 \Phi} \hat{\Phi}_{1+3}+(1+\alpha) \frac{\gamma_{1+3}}{2} \delta \widetilde{r} \\
& =\left(15+8 \gamma_{1+3}\right)(1+\alpha) \widehat{\omega}+3 \widehat{\omega}=(15+8 \bar{\gamma})(1+\alpha) \widehat{\omega}+3 \widehat{\omega}
\end{aligned}
$$

Savings bank 2 closes $\left((6 \omega+9 \alpha \omega)-(1+\alpha) \frac{\delta \widetilde{r}}{4 \Phi}\left(2-\gamma_{1+3}\right) \gamma_{1+3}\right)$ branches (at $\Phi$ units per branch), and, in each territory, gets a share $\left(2-\gamma_{1+3}\right) / 2$ of the market. ${ }^{28}$ Hence, its payoff is

$$
\begin{aligned}
\pi_{2} & =\left((6+9 \alpha) \frac{\delta \widetilde{r}}{32 \Phi} \Phi-(1+\alpha)\left(2-\gamma_{1+3}\right) \gamma_{1+3} \frac{\delta \tilde{r}}{4 \Phi} \Phi\right)+(1+\alpha) \frac{2-\gamma_{1+3}}{2} \delta \widetilde{r} \\
& =\left(41-32 \gamma_{1+3}+8 \gamma_{1+3}^{2}\right)(1+\alpha) \widehat{\omega}-3 \widehat{\omega}=\left(41-32 \bar{\gamma}+8 \bar{\gamma}^{2}\right)(1+\alpha) \widehat{\omega}-3 \widehat{\omega}
\end{aligned}
$$

- $(2+3,1)$ : The multimarket bank and local bank 2 merge with each other.

The analysis is analogous to that of the previous case. We obtain

$$
\begin{aligned}
\pi_{2+3} & =\left(15+8 \gamma_{2+3}\right)(1+\alpha) \widehat{\omega}+3 \alpha \widehat{\omega}=(15+8 \rho \bar{\gamma})(1+\alpha) \widehat{\omega}+3 \alpha \widehat{\omega} \\
\pi_{1} & =\left(41-32 \gamma_{2+3}+8 \gamma_{2+3}^{2}\right)(1+\alpha) \widehat{\omega}-3 \alpha \widehat{\omega} \\
& =\left(41-32 \rho \bar{\gamma}+8(\rho \bar{\gamma})^{2}\right)(1+\alpha) \widehat{\omega}-3 \alpha \widehat{\omega}
\end{aligned}
$$

\section{References}

Allen F, Gale D (2000) Comparing financial systems. MIT Press, Cambridge

Allen F, Gale D (2004) Competition and financial stability. J Money Credit Banking 36(3Pt.2):433-480

Amel DF, Liang NJ (1992) The relationship between entry into banking markets and changes in legal restrictions on entry. Antitrust Bull 37:631-649

Beck T, Demirguc-Kunt A, Levine R (2006) Bank concentration, competition, and crises: First results. J Banking Finance 30(5):1581-1603

Besanko D, Thakor AV (1993) Relationship banking, deposit insurance and bank portfolio. In: Mayer C, Vives X (eds) Capital markets and financial intermediation. Cambridge University Press, Cambridge pp 292-318

Berger A, Demsetz R, Strahan P (1999) The consolidation of the financial services industry: causes, consequences, and implications for the future. J Banking Finance, pp 135-194

Boot AW, Greenbaum S (1993) Bank regulation, reputation, and rents: Theory and policy implications. In: Mayer C. Vives X (eds) Capital markets and financial intermediation. Cambridge University Press, Cambridge, pp 292-318

Calem P (1994) The impact of geographic deregulation on small banks. Business Rev. Federal Reserve Bank of Philadelphia, Philadelphia, pp 17-31

Calomiris CW (2000) US bank deregulation in historical perspective. Cambridge University Press, New York

Caminal R, Gual J, Vives X (1989) Competition in Spanish banking. CEPR Discussion Paper no. 314. Centre for Economic Policy Research, London

Canals J (1997) Universal banking. Oxford University Press, Oxford

\footnotetext{
$\overline{28}$ Note that, for low $\gamma_{1+3}$ and high $\alpha,(6 \omega+9 \alpha \omega)-(1+\alpha) \frac{\delta \widetilde{r}}{4 \Phi}\left(2-\gamma_{1+3}\right) \gamma_{1+3}$ can be negative. In such a case, bank 2 opens branches.
} 
Carbó S (2004) Diez hechos estilizados del sector bancario español. Papeles de Economía Española 100:232-245

Cuñat V, Garicano L (2009) Did good cajas extend bad loans?: governance, human capital and loan portfolios. In: Bentolila S, Boldrin M, Díaz Jiménez J, Dolado JJ (eds) The crisis of the Spanish Economy (forthcoming)

Dermine J (1999) The economies of bank mergers in the European Union: a review of the public policy issues, WP, INSEAD

Economides N, Hubbard RG, Palia D (1996) The political economy of branching restrictions and deposit insurance: A model of monopolistic competition among small and large banks. J Law Econ 39:667-704

Fuentelsaz L, Gómez J, Lucea V (2006) Do multimarket banks and local banks, and credit unions compete? Int J Bus Econ 5(1):17-27

Gorton G, Kahl M, Rosen RJ (2009) Eat or be eaten: a theory of mergers and firm sizes. J Finance 44: 1291-1344

Gual J, Neven D (1992) Deregulation of the European banking industry (1980-1991), Discussion paper 703. CEPR, London

Hellman T, Murdock K, Stiglitz JE (2000) Liberalization, moral hazard in banking and prudential regulation: Are capital controls enough? Am Econ Rev 90(1):147-165

Jayaratne J, Strahan PE (1996) The finance-growth nexus: evidence from bank branch deregulation. Quart J Econ 111:639-670

Jayaratne J, Strahan PE (1998) Entry restrictions, industry evolution, and dynamic efficiency: Evidence from multimarket banking. J Law Econ 41:239-273

Kroszner RS, Strahan PE (1999) What drives deregulation? Economics and politics of the relaxation of bank branching restrictions. Quart J Econ 114:1437-1467

Lozano-Vivas A, Meléndez-Jiménez MA, Morales AJ (2010a) Branching deregulation and merger optimality. Málaga Economic Theory Research Center Working Papers 2010-2

Lozano-Vivas A, Meléndez-Jiménez MA, Morales AJ (2010b) Whom to merge with? A tale of the Spanish banking deregulation process. Málaga Economic Theory Research Center Working Papers 2010-3

Matutes C, Vives X (2000) Imperfect competition, risk taking and regulation in banking. Eur Econ Rev 44:184-216

McLaughlin S (1995) The impact of interstate banking and branching reform: evidence from the state. Current Issues in Economics and Finance. Federal Reserve Bank of New York, vol 1. No. 2

Piloff SJ, Santomero AM (1998) The value effects of bank merges and acquisitions. In: Amihud Y, Miller G (eds) Bank Mergers \& Acquisitions, Kluwer Academic Publishers, Dordrecht, pp 59-78

Qiu LD, Zhou W (2007) Merger waves: a model of endogenous mergers. Rand J Econ 38(1):214-226

Salas V, Saurinas J (2003) Deregulation, market power and risk behaviour in Spanish banks. Eur Econ Rev 47:1061-1075

Salop S (1979) Monopolistic competition with outside goods. Bell J Econ 10:141-156

Stiroh KJ, Strahan PE (2003) Competitive dynamics of deregulation: Evidence from U.S. banking. J Money Credit Banking 35:801-828

Tullock G (1980) Efficient rent-seeking. In: Buchanan J, Tollison R, Tullock G (eds) Toward a theory of the rent-seeking society. Texas A\&M Press, College Station, pp 97-112 